\title{
REFLEXIONES SOBRE LA ADMINISTRACIÓN PÚBLICA Y EL NEOLIBERALISMO EN NUESTRAMÉRICA, SIGLO XXI ${ }^{1}$
}

\author{
Paulo Ricardo Zilio Abdala ${ }^{2}$ \\ José Francisco Puello-Socarrás ${ }^{3}$
}

http://dx.doi.org/10.1590/1413-2311.247.94991

\begin{abstract}
El intento (la necesidad) de definir el ámbito histórico propio de nuestra América va acompañado, como es habitual en casos similares, por la búsqueda de la deno minación que mejor corresponda a ese ámbito; esa de nominación contribuye a mostrar el grado de conciencia que se tiene de aquello que se aspira a aprehender.
\end{abstract}

Roberto Fernandéz Retamar ${ }^{4}$

\section{RESUMEN}

En este ensayo argumentamos que solamente sería posible comprender las transformaciones de la administración pública en la actualidad a partir de una observación atenta sobre el desarrollo del capitalismo en distintos momentos de su etapa neoliberal, su historia y sus

\footnotetext{
${ }^{1}$ Recebido em 14/07/2019; aprovado em 05/08/2019.

${ }^{2}$ Universidade Federal do Rio Grande do Sul - Escola de Administração e PPGA; Porto Alegre - RS (Brasil) http://orcid.org/0000-0002-1977-8424. E-mail: paulo.abdala@ufrgs.br.

${ }^{3}$ Escuela Superior de Administración Pública; Bogotá (Colombia) - http://orcid.org/0000-0002-8658-9543. Email: josepuel@esap.edu.co.

${ }^{4}$ La expresión Nuestramérica está inspirada en Retamar, también como una forma de homenaje a este poeta, intelectual y militante cubano, figura central en el pensamiento social latinoamericano, recientemente fallecido, en julio de 2019, a la edad de 89 años.
} 
implicaciones teóricas y prácticas. En esa medida, problematizamos las transformaciones del neoliberalismo y su nueva versión en el siglo XXI, entre otros: el tránsito del principio antropológico desde el Homo Economicus hacia el Homo Redemptoris, el hombre emprendedor, a partir de la incorporación de nuevas matrices teóricas. Este redireccionamiento hace parte de una serie de transformaciones que se insinúan en relación al nuevo papel que cumpliría el Estado como "emprendedor" como facilitador de negocios; en la redefinición de la función de Gobierno, la cual tiende hacia la Gobernanza como forma política central; en la creación de los espacios públicos no estatales, con la regresión de las políticas sociales y el avance de "medidas" que giran hacia una supuesta libertad económica; y en la Administración Pública, comprendida como simple gestora de redes de contratos promovidos por los gobiernos en función de la iniciativa privada y los mercados y en los cuales se busca garantizar la eficiencia y la eficacia de los mismos, es decir, situaciones de lucro en particular y de la acumulación de capital en general. Esta impronta característica en el Estado neoliberal contemporáneo pretenden dar continuidad a la construcción estratégica de una sociedad de mercado para el siglo XXI, con drásticas implicaciones para los países dependientes, especialmente considerando la realidad de la super-explotación, miseria y de la desigualdad en América Latina y el Caribe “desde abajo”, es decir, de Nuestramérica.

PALABRAS CLAVE: Administración Pública. Neoliberalismo. Emprendimiento. Estado. Gobierno. Mercado.

\section{RESUMO}

Argumentamos neste ensaio que somente seria possível compreender as transformações da administração pública na atualidade a partir de um olhar atento para o desenvolvimento do capitalismo em distintos momentos de sua etapa neoliberal, com sua história e implicações teóricas e práticas. Para o desenvolvimento do argumento, problematizamos as transformações do neoliberalismo em sua nova versão no Século XXI, com a mudança do princípio antropológico do Homo Economicus para o Homo Redemptoris, ou homem empreendedor, a partir da incorporação de novas matrizes teóricas. Tal redirecionamento faz parte de uma série de transformações que se insinuam: em relação ao novo papel que deveria cumprir o Estado "empreendedor", como facilitador de negócios; na redefinição da função de Governo, a qual tende a Governança como forma política central; na criação de espaços públicos não estatais, com a regressão de políticas sociais e o avanço de "medidas" que se 
direcionam a uma suposta liberdade econômica; e na Administração Pública, compreendida como simples gestora da rede de contratos promovidos pelos governos em função da iniciativa privada e dos mercados e nos quais se busca garantir eficiência e eficácia, quer dizer, situações de lucro em particular e de acumulação de capital em geral. Esses traços característicos do Estado neoliberal contemporâneo pretendem dar continuidade à construção estratégica de uma sociedade de mercado para o Século XXI, com drásticas implicações éticas para os países dependentes, especialmente considerando a realidade da superexploração, da miséria e da desigualdade da América Latina e do Caribe "desde baixo", quer dizer, de Nuestramérica.

Palavras-Chave: Administração Pública. Neoliberalismo. Empreendedorismo. Estado. Governo. Mercado.

\begin{abstract}
We argue in this essay that it would only be possible to understand the transformations of public administration today from a close look at the development of capitalism at different times of its neoliberal stage, with its history and theoretical and practical implications. For the development of the argument, we problematized the transformations of neoliberalism in its new version in the 21st Century, with the shift from the anthropological principle of Homo Economicus to Homo Redemptoris, or entrepreneurial man, by incorporating new theoretical matrices. Such redirection is part of a series of transformations that are insinuated: regarding the new role that the "entrepreneurial" State should fulfill, as a business facilitator; in the redefinition of the function of Government, which tends to Governance as a central political form; in the creation of non-state public spaces, with the regression of social policies and the advancement of "measures" that address supposed economic freedom; and in Public Administration, understood as a simple manager of contracts networks promoted by governments as a result of private initiative and markets, which seeks to ensure efficiency and effectiveness, that is, situations of profit in particular and accumulation of capital in general. These characteristic traits of the contemporary neoliberal State are intended to continue the strategic construction of a 21 st century market society, with drastic ethical implications for dependent countries, especially considering the reality of overexploitation, misery and inequality in Latin America and the Caribbean. "From below", that is, from Nuestramérica.
\end{abstract}

REAd | Porto Alegre - Vol. 25 - No 2 - Maio / Agosto 2019 - p. 22-39 
KEYWORDS: Public Administration. Neoliberalism. Entrepreneurship. State. Government. Market.

Estudiar la relación entre el neoliberalismo y la administración pública es fundamental para comprender los drásticos cambios de nuestro tiempo. El neoliberalismo como etapa superior del capitalismo, evidencia (teórica, empírica e, inclusive, estadísticamente) la exacerbación de sus principales dinámicas, lógicas y contradicciones, movilizando fuerzas culturales, políticas y sociales que alteran la manera cómo los gobiernos y los Estados resultan ser administrados. Los cambios en curso, acompañados por la profundización de un nuevo ciclo de reformas del aparato de Estado, están marcadas por cuestiones de fondo que y muestran una fuerte continuidad desde -al menos- los años 1980s. Aunque sus modus operandi parezcan ser "inéditos", en realidad, son fruto de la capacidad adaptativa del neoliberalismo (una singular capacidad de resiliencia que generalmente se omite en los análisis), sus teorías y sus prácticas, en diferentes momentos históricos del capitalismo.

En la actualidad, la tendencia global calcada por las máximas del conservadorismo en los valores y el (neo)liberalismo en la economía viene siendo responsable del impulso (contra)reformador ofensivo por parte del Capital contra todos los derechos sociales históricamente logrados. El papel del Estado está siendo -una vez más- redefinido, respaldando una nueva forma de administración pública alineada ( $\mathrm{y}$, por qué no decirlo también: alienada) con una modalidad renovada de neoliberalismo que se muestra más feroz y agresiva que en contraste y comparación con sus versiones antecesoras.

El argumento de este ensayo parte de la idea que solamente es posible comprender las transformaciones de la administración pública en la actualidad a partir de una aproximación atenta sobre la evolución del capitalismo en los distintos momentos de su etapa neoliberal, teniendo presente no sólo su historia sino también sus implicaciones teóricas y prácticas.

Aunque el tema sea de por sí esencial, frecuentemente se encuentra relegado a un segundo plano. Destacadas contribuciones académicas de reciente publicación y que compilan diferentes reflexiones y perspectivas analíticas sobre el neoliberalismo (The SAGE Handbook of Neoliberalism, un texto de 682 páginas!, es uno de los ejemplos), apenas registran sistemáticamente temas, tópicos, efectos o implicaciones del neoliberalismo en términos de la administración pública como fenómeno y campo del saber (CAHILL; COOPER; KONINGS; PRIMROSE, 2018). El libro en mención, en formato de un mosaico 
del conocimiento poco contribuye para la profundización del tema, reforzando la idea que el neoliberalismo puede ser comprendido a partir de múltiples perspectivas, como si fuese un fenómeno interpretativo y no histórico, al mejor estilo post-estructuralista.

Más específicamente, el Handbook aborda ocasionalmente fenómenos relativos a la administración pública que los confina -en la práctica- a un capítulo específico sobre la ascensión de Tony Blair y Bill Clinton como Tercera vía política en los orígenes en una Segunda onda expansiva del neoliberalismo después de Thatcher y Reagan. Se omite así el papel geopolítico clave de América Latina y el Caribe en tanto periferia capitalista dentro del "nacimiento" y la posterior evolución y renovación neoliberal a nivel global.

La poca importancia dada a la relación entre administración pública y neoliberalismo puede ser explicada, al menos en parte, por la marca de nacimiento de la Administración pública basada en la falaz separación entre técnica y política (GUERRERO, 1980), matriz que perdura hasta el día de hoy. Esa brecha creada artificialmente, por un lado, explica la facilidad en la incorporación del gerencialismo dentro del cuerpo empírico-teórico de la administración pública contemporánea (MISOCZKY, 2004), ya que los acontecimientos derivados de la teoría general de la administración no son ajenos a su constitución; por otro, obstruye y evita el diálogo con el pensamiento crítico, al limitar la administración pública a un conjunto de técnicas de gestión. Aunque se defienda la utilización e investigación de técnicas específicas para la gestión de la "cosa pública", la delimitación de fronteras excluyentes sobre una comprensión más amplia del papel de la Administración pública en el contexto del desarrollo del capitalismo reduce la capacidad de reflexión sobre el saber público-administrativo.

La separación entre técnica y política ha llevado los estudios del campo de la administración pública a subestimar la dimensión analítica y privilegiar trabajos prescriptivos y normativos que, en general, apuntan a comprensiones y explicaciones de las realidades gubernamentales emergentes, limitándose a describirlas (FADUL SILVA; SILVA, 2012). Tal hipótesis fue corroborada en Brasil a partir de la investigación de Corrêa (2017, p. 158) sobre la historia del campo de conocimiento de la administración pública. Según indican los resultados de la investigación, el foco predominante sigue concentrado en un paradigma funcionalista, y así bastante menos atento con los desarrollos de orden teórico. De manera complementaria, las técnicas empleadas son rehenes de análisis erráticos y modismos relacionados con supuestos cambios de paradigma, avanzando muy poco dentro de una reflexión íntegra, compleja y más completa de la administración pública. 
Estos puntos de vista funcionalista en el área han conducido, por demás, hacia indagaciones desde un sentido común distorsionado frente a cuestiones fundamentales como el neoliberalismo. Aunque su efecto práctico sea contundente y su mención habitual, especialmente cuando las reformas gerenciales están bajo lupa, se trata de un fenómeno, en general, mal comprendido, habiendo poca claridad sobre su concepto, límites y consecuencias. La explicación para esa ignorancia reside, en parte, en la amplia gama de fenómenos sociales, políticos y económicos, de variados grados de complejidad y abstracción, que son relacionados con las modalidades de neoliberalismo sin rigor teórico (SAAD-FILHO; JOHNSTON, 2005).

No obstante, a pesar de la diversidad de sus manifestaciones, el neoliberalismo exhibe regularidades que se generalizan en diversos contextos y experiencias históricas. Según Harvey (2005, p.2), “el neoliberalismo es, en primera instancia, una teoría sobre las prácticas de la política económica que propone que el bienestar humano puede ser mejorado a través de la capacidad individual de las libertades y habilidades emprendedoras, dentro de un cuadro institucional caracterizado por el fortalecimiento de los derechos a la propiedad privada, libertad de mercados y de comercio". En ese contexto, el papel preponderante del Estado es crear y preservar un ambiente propicio para que las prácticas de negocios se desarrollen y se consoliden en términos de clase. En otras palabras: existe una ocultación sistemática de la explotación de los trabajadores a través de la dominación política y de las opresiones sociales en función del "libre desarrollo del Capital" - según decía el reconocido intelectual neoliberal austríaco Ludwig Von Mises - y su acumulación incesante.

La Administración pública, justamente, se constituye a través de estructuras y prácticas por medio de las cuales las orientaciones neoliberales vienen siendo materializadas en formaciones económico-sociales específicas. Si el pragmatismo funcionalista es lo que impera en el campo, no es de esperar otra cosa sino la reproducción del cuadro institucional con sus apelaciones a la eficiencia y la modernización para la administración pública en América Latina y el Caribe.

Para que esas cuestiones fundamentales sean tratadas con la debida profundidad, es necesario vincular nuevamente administración y política, rechazando las perspectivas tecnicistas, descriptivistas y -supuestamente- neutras, las cuales deben ser cuestionadas por sus incapacidades analítica, explicativa y/o reflexiva. Entendiendo que la administración pública aborda la relación entre el Estado y la sociedad, con base en la acción (o inacción, incluso contemplando la "no-acción”) gubernamental (GUERRERO, 1980), su campo de 
estudios debe ser abordado sobre la perspectiva de las relaciones de poder, intentando, más específicamente, su implicación en el dominio político y en la acción administrativa.

El neoliberalismo, tal como se sostiene en este ensayo, según Puello-Socarrás (2008), no se limita a un conjunto (o programa) de políticas, siendo mejor comprendido como la actual etapa del capitalismo. Uno de los equívocos comunes al tratar el tema es agotarlo en el tristemente célebre Consenso(s) de Washington, instalando comprensiones superficiales que enredan su significado político y económico. Un ejemplo de cómo esa miopía puede causar distorsiones es la interpretación sobre la orientación política en el liderazgo de los Estados nacionales latinoamericanos en tiempos recientes. La ausencia de referencias históricoestructurantes frecuentemente conduce hacia interpretaciones que sobreestiman las posibilidades de cambios a partir de los gobiernos. El neoliberalismo no se explica entonces como una cuestión de adhesión por decisión política. Aún así, es común encontrar una corriente de análisis -moralmente favorable- sobre los efectos de la llamada Ola progresista latinoamericana de la primera década de los años 2000 como supuesta "ruptura antineoliberal", sin profundizar debidamente sobre la importancia en los cambios del neoliberalismo hacia una nueva versión (ABDALA; CAMARA, 2015; PUELLOSOCARRÁS, 2013).

El neoliberalismo, como dinámica actualizada del desarrollo capitalista, viene presentando diferentes facetas, incorporando nuevas referencias sin modificar sus contenidos centrales. La estrategia sigue siendo crear nuevos espacios para la acumulación incesante del capital como un proyecto de clase (capitalista transnacional) para combatir las crisis constantes del capitalismo. Las expresiones tácticas del neoliberalismo varían en cada momento histórico, socavando las resistencias a su proyecto y totalizando e "innovando" nuevas dinámicas y repertorios para seguir consolidando su hegemonía como alternativa única. Rememorando la famosa frase, frecuentemente utilizada como lema por los gobernantes al defender (contra)reformas: no hay alternativa! (el acrónimo: TINA, there is no alternative).

Como explica Puello-Socarrás (2013), el neoliberalismo no se basa en un pensamiento monolítico sino que cuenta con variaciones ideológicas fruto de distintas corrientes teóricas en sus marcos. La transición del neoliberalismo hacia el nuevo neoliberalismo durante el siglo XXI se basa en una (relativa) renovación de las perspectivas ideológicas y práxis que lo respaldan como proyecto político de clase (transnacional). Uno de los signos más reveladores de su nueva faceta ha sido la preeminencia que adquieren, al nivel de las teorías sociales en general y en las teorías económicas en particular, las perspectivas consideradas subsidiarias 
dentro del neoliberalismo durante el siglo anterior (austríacas, alemanas e italianas) frente a la hegemonía de las posturas angloamericanas. No entraremos aquí en los detalles de cada una de las vertientes, pero, a su manera, sobre ellas se encuentra fundamentada la renovación del neoliberalismo a nivel global en el siglo XXI.

Uno de los principales indicios de esta nueva época estaría marcado por un cambio ontológico crucial. Mientras que las corrientes Anglo-Americanas mantenían como principio antropológico al Homo Economicus, el individuo racional y calculador -próximo a la figura destacada por el pensamiento de la economía política liberal clásica -, las corrientes austríacas, alemanas e italianas vienen elevando al Homo Redemptoris, el hombre emprendedor, "empresario de sí mismo" (aunque no para sí mismo sino para la acumulación renovada del Capital), como protagonista del capitalismo del nuevo milenio. El hombre emprendedor es el sujeto social neoliberal por antonomasia. Él es el responsable de guiar la producción social a partir de la coordinación "espontánea" dentro de la división social del trabajo. No solamente a través de la creación de empresas y por medio de los procesos de innovación ya existentes -como generalmente se subraya en los discursos sobre emprendimiento- sino, además de eso, provocando la empresarialización de las relaciones sociales, empezando por los individuos.

El desplazamiento del principio ontológico del nuevo neoliberalismo, desde luego, no llega a ser una ruptura, ya que las diferentes perspectivas convergen en la práctica, superando estratégicamente sus diferencias en una unidad ideológica. Tal modificación es, en realidad, una adaptación que permite colocar al empresario/emprendedor como fuerza fundamental (no sólo una estructura mercantil) de la sociedad de mercado contemporánea. Para hacerlo inteligible, Amazon es una empresa y, simultáneamente, un mercado.

Por esta razón, la reintegración de las visiones "centradas en el empredimiento" y la valoración del "espíritu emprendedor" a lo largo del siglo XXI se tornan cruciales para el grado de recomposición social de la crisis del capitalismo neoliberal, gracias a la productividad reportada por el discurso emprendedor, especialmente en los procesos de alineación ideológica. Las distintas modalidades de auto-empleo, trabajo autónomo, free lance, y todos los tipos de ocupaciones de la economía "creativa", incluyendo el fenómeno de las starts-up traen de suyo la promesa de nuevas soluciones para amortiguar las agudas contradicciones actuales entre Capital y Trabajo. A partir de esa suposición, varios teóricos neoliberales contemporáneos han predicho la inminente transición hacia una sociedad postcapitalista (por ejemplo, RIFKIN, 1994). La euforia emprendedora ha llegado hasta el punto que ciertos sectores de la izquierda intelectual euroamericana enarbolen vías hacia la 
emancipación post-capitalista desde el emprendimiento de la multitud, una suerte de emprendimiento "anti-capitalista" (!a partir de la yuxtaposición entre J. Schumpeter y K. Marx!), tal y como reza un capítulo dedicado a este tema en Assembly (HARDT; NEGRI, 2017).

Las experiencias concretas del emprendimiento a nivel global, entre tanto, muestran todo lo contrario. Antes que verificar cualquier ruptura (así sea moderada) en las tendencias de los procesos de explotación económica, dominación política y opresión social, los resultados revelados por las iniciativas emprendedoras registran mayores niveles de explotación, especialmente de subordinación y dependencia de los emprendedores al capital financiero, vía endeudamiento progresivo; y la precariedad de las situaciones de trabajo incluso de aquellas autoimpuestas. Para tener una idea de la dimensión del problema, datos del SEBRAE de 2018 indican que el 37,5 de los nuevos negocios en Brasil fueron motivados por necesidad, algo próximo a 9 millones de emprendimientos (GEM, 2018). En Colombia, por su parte, un estudio de la Red de Cámaras de Comercio reveló que solo entre 2011 y 2015 "entraron al mercado un total de 1.033.211 firmas y se cancelaron 991.911" (CONFECAMARAS, 2016: 21), lo que significa que "en el neto hay una mortalidad del 96\%. Ello hace referencia a empresas formales de todas las clases, pero en los sectores populares... es lo que se conoce como "emprendimiento de subsistencia", el fracaso ronda el 97\%" (GIRALDO, 2017). No se trata, por lo tanto, de un cambio de mentalidad sino de condiciones materiales que se imponen a los que antes trabajaban contratados, recibiendo protección social mínima, ahora lanzados hacia una condición de emprendedores forzados.

Se establece, así, una nueva relación entre el emprendedor "consigo mismo" de un lado (auto-explotación, auto-subordinación, etc.), y el sector financiero del otro, mediador del capital que siempre carecen los "trabajadores de sí mismos" para activar y sustentar su factea emprendedora. Es crucial, por tanto, entender cómo la hegemonía neoliberal emprendedora exacerba los procesos de mercantilización en todas las dimensiones y esferas de la vida humana, individual y colectivamente consideradas. Casilli (2018, en línea), lo sintetiza de la siguiente forma:

[...] el capitalismo de las plataformas digitales hace que la disciplina laboral sea más rígida, ya que impone mediciones "científicas" y evaluaciones que 'pueden parecerse a los de la vieja fabricación industrial. La diferencia clave es que los trabajadores, a cambio de su sumisión a esta disciplina, no reciben la seguridad social y la representación política que tenían a cambio de su subordinación. Este 
nuevo taylorismo tiene todos los inconvenientes y ninguno de los antiguos beneficios. Los trabajadores están atrapados dentro de una contradicción: subordinados y precarios, al mismo tiempo.

En todo caso, estos cambios no se limitan al nivel de los individuos sino que adquieren una dimensión social. Sirven como soporte para una transición desde las políticas volcadas al trabajo hacia las políticas que enfatizan una supuesta autonomía y el derecho a vender con la "mayor libertad posible" la fuerza de trabajo en el mercado bajo las condiciones de la economía política actual. Esto justifica toda una serie de reformas que flexibilizan el trabajo y, más recientemente, llevan al reconocimiento y aceptación de los negocios de plataforma, en detrimento de antiguas formas de concesión y regulación de los servicios por el Estado como domicilios, taxis, localización de vehículos, concesión de canales de televisión y radio, derechos de autor en la música, entre otros.

Otro rasgo que se impone para el neoliberalismo actual es la matización de su acento, en principio fuertemente económico, hacia perspectivas más amplias y preocupadas por cuestiones culturales, sociales e institucionales. Como respuesta a la crisis de legitimidad ocurrida a mediados de la década de 1990, y que se profundiza espectacularmente desde el choque de 2007/2008, en el cual el sistema financiero y sus tentáculos fueron duramente criticados por movimientos de ocupación en diferentes lugares del planeta, ahora se pone sobre la mesa una opción de reconciliación, o como propone Stolowicz (2016), de "estabilización" neoliberal.

Revestido como un tercer lugar epistémico, supuestamente más allá de la dicotomía Estad-Mercado, el nuevo dominio social del nuevo neoliberalismo desvincula lo público de lo estatal. Recrea así un espacio público "no estatal" que resulta, fundamentalmente, una mercantilización exacerbada, ambiente perfecto para la proliferación del emprendimiento y sus desdoblamientos (recordamos que la teoría del emprendedor, lo propone como un "tercer" actor más allá del Trabajador y del Capitalista). Este rasgo tiene profundas implicaciones en las más diversas esferas de la vida en sociedad, provocando "una transición desde la conceptualización de los derechos del ciudadano del Estado de Derecho ("obsoleto") hacia la prestación de servicios sociales simples (como son llamados ahora en el "nuevo" Estado Gerencial) (PUELLO-SOCARRÁS, 2018a, p. 17). El individuo no es más visto como un ciudadano, siendo mejor entendido como un cliente y, más recientemente, como un usuario a ser satisfecho en sus necesidades no por el Estado sino por medio de los mecanismos de mercado, aunque muchas veces la empresa prestadora de los servicios esté actuando en una función pública. 
Las consecuencias para la administración pública de esta transición son muchas y variadas y marcan su evolución reciente como teoría y práctica. Esas relaciones se expresan a través de múltiples vínculos operacionales y conexiones teóricas, desde el gerencialismo de la Nueva Gestión Pública hasta, más recientemente, la "crítica domésticada" (MISOCZKY, 2010) que se origina con la "Pos-nueva" Gestión Pública (LÊGREID, 2017) y las teorías del Valor público (BRYSON; CROSBY; BLOOMBERG, 2014).

La reconstrucción del proyecto político de clase neoliberal, tanto al nivel del Estado político como de sus aparatos post-burocráticos actualmente vigentes, implica una constitución gradual pero sostenida del llamado Estado emprendedor -siendo Mazzucato (2014) una de sus promotoras más reconocidas.

En términos teóricos esta realidad emergente significa:

(i) Un nuevo tipo de configuración estatal neoliberal al nivel de la formalidad legal-jurídica y su realización específíca en términos de acción estatal y material, en el sentido de las relaciones sociales. El Estado no apoya exclusiva y directamente al emprendedor (individualmente considerado) sino que reorganiza sus estructuras (con base en los aparatos estatales post-burocráticos) y funciones (acciones generalmente de baja intensidad, las cuales más que "políticas" stricto sensu, son simples "medidas" públicas) con el objetivo de apoyar la relación (capitalista) emprendedora. En ese escenario sociopolítico, es posible, por un lado, regular (aunque no "intervenir") y estabilizar los conflictos sociales, "gerenciando" tácticamente los contextos de creciente inseguridad social, acentuados por el propio neoliberalismo.

(ii) En términos organizacionales, la profundización de las lógicas en dirección hacia la ("Post-nueva") Gestión del Valor público, las cuales tienden a funcionar cada vez más a partir de la centralidad de la gestión contractual -no solamente "hacia afuera" (outsourcing) sino también "hacia adentro" (insourcing) del Estado- y la flexibilización de los aparatos burocráticos y cada vez menos mediante mandatos público administrativos, los cuales tienden a funcionar marginalmente $\mathrm{o}$, al menos, sin la centralidad que tuvieron en los arreglos de la Administración pública durante el siglo XX. Aunque recientemente vienen difundiéndose críticas a las modalidades de la Nueva Gestión Pública por parte de corrientes como la (supuesta) "Post-nueva" Gestión pública y las aproximaciones sobre "Valor Público", incluído la emergente Whole-of-Government approach (CHRISTENSEN, 2012; CHRISTENSEN; LÆGREID, 2013, 2007a, 2007b, 2005; CHICA; SALAZAR, 2016), el reemplazo de las “antiguas" E's (eficiencia, eficacia, efectividad) por las “nuevas" C's (confianza, co-responsabilidad, concurrencia, colaboración, co-operación, comunicación, co- 
ordinación) no significan ruptura alguna. Por el contrario, se trata de la profundización actualizada de las praxis ideológicas organizacionales para la ("nueva") realidad y estructura de los campos post-burocráticos público-privados en la construcción histórica del neoliberalismo del nuevo milenio.

(iii) A partir de las dinámicas gubernamentales, la introducción e institucionalización de la Gobernanza como fórmula política del gobierno contemporáneo, dispuesta para las lógicas de "co-ordenación + co-operación" de los mecanismos de mercado (que hoy se muestran insuficientes, o en palabras coloquiales: no funcionan apropiadamente, dando paso a las "fallas de mercado", que justifican la regulación estatal oportunista e intermitente) respalda por el Estado en su papel primordial de "hacer cumplir los contratos" (rule of law). Así, la principal función del Estado emprendedor - propone Mazzucato (2011, p. 16): “has not just fixed markets but actively created them" ("no sólo corregir los mercados sino activamente crearlos").

Estos trazos característicos del Estado neoliberal contemporáneo pretenden dar continuidad a la construcción estratégica de una Sociedad de Mercado para el siglo XXI (y no sólo de una "economía" de mercado), si bien en medio de un cuadro general que muestra la profundización de la crisis capitalista global, la cual introduce grados más elevados de contradicciones e incertidumbres.

En términos prácticos, las reformas administrativas y de la gestión pública son expresión de las contradicciones de ese proceso, encontrándose marcadas por continuidades y rupturas que permiten la adaptación de los aparatos administrativos, estatales y no estatales, y de los esquemas de gestión en función de las necesidades tácticas y estratégicas de la etapa neoliberal del desarrollo capitalista ((MISOCZKY; ABDALA; DAMBORIARENA, 2017). Así las cosas, las reformas de la administración pública lejos de ser eventos coyunturales, forzados por las circunstancias geopolíticas particulares; son un proceso continuo que abre nuevos espacios para la acumulación de Capital.

La Administración pública bajo esta versión debería ser comprendida progresivamente como una estructura gestora de redes de contratos no gubernamentales respaldados funcionalmente por gobiernos estatales, con el propósito de garantizar la eficiencia y eficacia, de los mismos. Una de las expresiones elocuentes de los nuevos principios operativos del Estado contractual empresarial (en síntesis: “emprendedor") se resume en el tránsito desde la privatización del siglo XX hacia la desprivatización del siglo XXI, la cual se concreta en diferentes instrumentos, entre los más conocidos: las alianzas público-privadas. Estas modalidades se tornan hegemónicas tanto nacional como 
internacionalmente. El mentado "retorno del Estado" que en diferentes oportunidades ha sido ocultado bajo suposiciones del regreso falaz de la estatización, se explica por la necesidad neoliberal de la regulación estatal, esto es: "Estado fuertes para Mercados libres", como se propuso originalmente por el pensador neoliberal alemán Wilhelm Röpke en la década de 1930. Este lema que también resume los cambios del periodo, hoy vigente, ha sido resucitado por el desarrollismo neoliberal latinoamericano del siglo XXI a partir de la máxima: "No hay mercados fuertes sin un Estado fuerte" (ver BRESSER-PEREIRA 2011).

Tal función del Estado resulta importante ya que facilita los negocios, actuando para corregir las "fallas" del mercado. Es decir, el Estado actúa por medio de su aparato de gestión para auxiliar a que los negocios funcionen y prosperen, creando un ambiente propicio para el lucro. Esto incluye diversas facilidades para empresas (especialmente transnacionales), a través de grandes reducciones y exenciones impositivas y, en el límite, políticas regresivas en materia de protección ecológica, disminución de las normas de seguridad, renegociaciones de deuda y facilidades de acceso a financiamientos e infraestructura.

Entre tanto, estos nuevos modos de regulación neoliberal no se limitan a los sectores económicos tradicionales. Se dirigen firmemente en dirección a la mercantilización de otras dimensiones de la vida social. Por ejemplo, la cultura en general y la propiedad comunal en particular han sido objeto de una creciente mercantilización. En este sentido, las visiones sobre el emprendimiento han desempeñado un papel fundamental en la recreación de “innovaciones" en estos campos. Es el caso de las llamadas "Industrias Culturales Creativas" (HOWKINS, 2001) en los países centrales, o para el caso de América Latina y el Caribe, alrededor de las llamadas "Economías Naranjas" (BUITRAGO; DUQUE, 2013), promovidas selectivamente por organizaciones financieras internacionales, como el Banco InterAmericano de Desarrollo (BID) y por gobiernos políticos de diferentes espectros ideológicos que elevan tales formatos hacia orientaciones en políticas públicas oficiales en diferentes países de la región. En el caso del actual gobierno colombiano, presidido por Iván Duque, se pretende que el Estado promueva la apertura y "conquista" de los mercados en función de los emprendedores con base en una nueva "economía" y tipos de "bienes y servicios" culturales (BUITRAGO; DUQUE 2013, p. 40).

EL despliegue de la Economía Cultural de las industrias creativas, como la Economía Naranja, pretende, por un lado, institucionalizar al nivel de las orientaciones políticas del Estado una fórmula de "bien-estar" dependiente y basada en el emprendimiento (recordando que el lema de este gobierno es: "legalidad, emprendimiento y equidad); y, por otro lado, al nivel de las medidas gubernamentales específicas, la progresiva comercialización de la 
herencia de los patrimonios culturales con fines lucrativos. En este, como en otros casos, los procesos de neoliberalización en curso también (re)colonizan la dimensión cultural, intensificando el despojo y la depredación de las producciones sociales y populares (ver PUELLO-SOCARRÁS, 2018a, 2018b).

Otro ejemplo reciente en Brasil: la Medida Provisoria de la Libertad Económica, aprobada en mayo de 2019 por el Gobierno Federal (BRASIL, 2019), ayudaría ilustrar las transformaciones que venimos discutiendo. El texto de la medida previene sobre la liberación total para las personas físicas y jurídicas para desarrollar negocios considerados de bajo riesgo, sin la necesidad de licencias, autorizaciones, inscripciones, registros o permisos. También se prevé la "inmunidad burocrática" en el desarrollo de nuevos productos y servicios en las start-up's, permitiendo diferentes "testeos" (pruebas experimentales) en grupos e individuos sin autorización previa.

En su resumen ejecutivo, esta medida se justifica por.

[...] la necesidad urgente de alejar la percepción de que en Brasil el ejercicio de actividades económicas depende de la previa autorización del Estado. Ese escenario dejaría al particular sin la seguridad para generar empleo y renta. De ahí ocurre el hecho que Brasil figure "en la posición 150 en la generación de empleo en el Heritage Foundation/Wall Street Journal, la posición $144^{\circ}$ en el ranking de Libertad Económica del Instituto Fraser, y la posición $123^{\circ}$ en el ranking de Libertad Económica y Personal del Instituto Cato”.

La libertad económica es fundamental para el desarrollo de un país, inclusive más en el caso de Brasil, actualmente hundido en una crisis económica. Estudios que involucran más de 100 países a partir de la segunda mitad del siglo XX revelan esa relación entre libertad económica y el progreso.

La medida provisoria empodera al particular y se eleva contra los excesos de la interención del Estado, con el prpósito de estimular el emprendimiento y el desarrollo económico." (BRASIL, 2019, online)

El texto deja en claro la orientación del Estado emprendedor, en el sentido de Mazzucato (2011), aplicada para una política pública. La justificación pretende apoyarse en argumentos bastante dudosos, como si la relación entre libertad económica y progreso fuese lineal y directa, además de no considerar las relaciones de poder entre países, por traer uno de tantos ejemplos reales. El pseudo-argumento expuesto por la medida, omite flagrantemente 
que fue a través de la colonización y de las relaciones de dependencia y de sobre-explotación que el Norte global logró “desarrollarse". El "progreso", en este caso, se obtuvo a costa de la ausencia de libertad de los pueblos esclavizados de las periferias del planeta, quienes pagaron con su vida el precio del desarrollo capitalista. Por tanto, la relación directa entre libertad económica y progreso resulta ser falaz, dejando de considerar a los países beneficiados por la distribución geopolítica de poder y evitando el análisis histórico entre los países explotados y explotadores y la inmensa contribución de los mal-llamados países "subdesarrollados" dentro de la construcción de la realidad actual. Esta verdad incontestable pero silenciada en el pensamiento convencional en general y por el pseudo-argumento anterior, puntualmente, la sintetiza Retamar (2006) cuando postula la inexistencia de países “desarrollados", por un lado y "subdesarrollados", por el otro; debe hablarse -si es el caso- de países subdesarrollados y países "subdesarrollantes" No será la libertad económica la que resolverá los problemas de pobreza y de miseria de América Latina y el Caribe en el contexto de sobre-explotación del trabajo. Lo que ocurrirá, de hecho, es la ampliación e intensificación de los ya conocidos, dramáticos e históricos problemas estructurales del capitalismo tardío que se profundizan con el doble giro anti-social y anti-popular que actualiza el nuevo neoliberalismo en el siglo XXI.

El proceso en cuestión, problematizado en este ensayo, aún es reciente e incierto en cuanto a sus desdoblamientos y límites. Lo cierto es que vivimos tiempos intensos de transformaciones que requieren la introducción de nuevos abordajes e instrumentos analíticos y teóricos en la investigación sobre la Administración pública. Como, entre otros, propone González Casanova (2017), ya no es posible ignorar más el contexto económico político e histórico del modo de producción capitalista que modifica las reglas de juego, imponiendo nuevos modos de acción a los que trabajan con la función pública. Considerando el papel que debe ser desempeñado por la academia, esto implica: (1) asumir críticamente las posiciones entusiastas (ingenuas) que defienden la idea que el Estado y los agentes públicos actúan en pro de una entelequia: el "bien común" debido a la posición estructural que ocupan en la división social del trabajo (tanto a nivel nacional como, más importante aún, a nivel inter- y trans- nacional); (2) fortalecer una agenda renovada de investigación que refleje las contradicciones y dificultades reales de la función pública en los tiempos actuales; (3) ampliar la discusión sobre la importancia de las luchas sociales por la manutención y ampliación de los servicios públicos en clave de derechos populares desde reflexiones ética en perspectiva de su razón de ser, especialmente considerando la realidad de la sobreexplotación, miseria y desigualdad registradas ayer y hoy en Nuestramérica. 


\section{REFERENCIAS}

ABDALA, P.; CÂMARA, G. O novo desenvolvimentismo como farsa e o novo neoliberalismo como fato: as 8 teses do novo neoliberalismo na realidade brasileira. Anuario em Estudios Politicos Latinoamericanos, vol. 2, nov., 2015, p. 97-125.

BRASIL. Sumário Executivo de Medida Provisória. Medida Provisória no 881, de 2019.

Disponível em: https://www.congressonacional.leg.br/materias/medidas-provisorias//mpv/136531. Acesso em: 24 jul. 2019.

BRESSER-PEREIRA, L.C. An account of new developmentalism and its structuralist macroeconomics. Brazilian Journal of Political Economy, v. 31, n. 3, p. 493-502, 2011. BRYSON, J. M.; CROSBY, B. C.; BLOOMBERG, L. Public value governance: Moving beyond traditional public administration and the new public management. Public Administration Review, v. 74, n. 4, p. 445-456, 2014.

BUITRAGO, F. e DUQUE, I. La Economía Naranja: una oportunidad infinita. Nueva York: Banco Interamericano de Desarrollo, 2013.

CAHILL, D.; COOPER, M.; KONINGS, M.; PRIMROSE, D. (eds.) The SAGE Handbook of Neoliberalism. Los Angeles: SAGE Reference, 2018.

CASILLI, A. "Los trabajadores son el corazón del algoritmo": El nuevo capitalismo digital. Entrevista. Sinpermiso. Disponível em: http://www.sinpermiso.info/textos/los-trabajadoresson-el-corazon-del-algoritmo-el-nuevo-capitalismo-digital-entrevista. Acesso em: 23 jul. 2019.

CONFECAMARAS. Nacimiento y supervivencia de las empresas en Colombia. Bogotá, Red de Cámaras, 2016. Disponível em: https://bit.ly/2DNBydT. Acesso em: 29 jul. 2019. CORRÊA, V. T. O campo do conhecimento em administração pública no Brasil: uma análise histórica a partir do seu contexto e caráter multifacetado. 2017. Tese (Doutorado em Administração de Organizações) - Faculdade de Economia, Administração e Contabilidade de Ribeirão Preto, Universidade de São Paulo, Ribeirão Preto, 2017.

CHICA, S., SALAZAR, C. Nueva y posnueva gestión pública ¿Continuidad o ruptura de las doctrinas de reforma a partir de 1990? Administración y Desarrollo, v. 46, n. 1, p. 100-125, 2016.

CHRISTENSEN, T. Post-NPM and changing public governance. Meiji Journal of Political Science and Economics, v.1, p. 1-11, 2012.

CHRISTENSEN, T.; LÆGREID, P. Transcending New Public Management: The

Transformation of Public Sector Reforms. Burlington: Ashgate Publishing Company, 2013. 
CHRISTENSEN, T.; LÆGREID, P. Reformas Post nueva gestión Pública. Tendencias empíricas y retos académicos. Gestión y Política Pública, v. XVI, n. 2, México, p. 539-564, 2007.

CHRISTENSEN, T.; LÆGREID, P. El estado fragmentado: los retos de combinar eficiencia, normas institucionales y democracia. Gestión y Política Pública, v. XIV, n. 3, p. 557-598, 2005.

FADUL, E. M.; SILVA, L. P. Retomando o debate sobre a Reforma do Estado e a Nova Administração Pública. Anais do XXXII Encontro da Anpad, 2008.

GEM. Global Entrepreneurship Monitor. Empreendedorismo no Brasil. Relatório Executivo. Paraná, 2018. Disponível em: http://datasebrae.com.br/wpcontent/uploads/2019/02/Relat\%C3\%B3rio-Executivo-Brasil-2018-v3-web.pdf. Acesso em: 30 jul. 2019.

GIRALDO, C. Va por buen camino la Reincorporación de las FARC? Palabras al Márgen (octubre). Disponible en: https://bit.ly/2fXWrN0. Aceso en: 24 ene. 2019.

GUERRERO, O. La Administración Pública del Estado Capitalista. Barcelona: Fontanara. 1980.

HARDT, M.; NEGRI, A. Assembly. Oxford: Oxford University Press, 2017.

HARVEY, D. Neoliberalism: a short history. Oxford: Oxford University Press, 2005.

HOWKINS, J. The Creative Economy: How people make money from ideas. London: Allen Lane, 2001.

LÊGREID, P. Transcending new public management: the transformation of public sector reforms. New York: Routledge, 2017.

MAZZUCATO, M. The entrepreneurial State. Demos: London, 2011.

MAZZUCATO, M. El Estado emprendedor: mitos del sector público frente al sector privado. Barcelona: RBA, 2014.

MISOCZKY, M.C.; ABDALA, P. R.; DAMBORIARENA, L. A. A trajetória Ininterrupta da Reforma do Aparelho de Estado no Brasil: Continuidades nos Marcos do Neoliberalismo e do Gerencialismo. Administração Pública e Gestão Social, v. 1, n. 3, p. 184-193, 2017. MISOCZKY, M.C. Administração Pública Contemporânea. Porto Alegre: Ministério da Cultura/UFRGS/EA, Módulo 4. Apostila do Curso de Extensão em Administração Pública da Cultura, 2004.

MISOCZKY, M.C. Das praticas não-gerenciais de organizar a organização para a práxis da libertação. In: MISOCZKY, M. C., KRUTER, R., e MORAES, J. Organização e práxis libertadora. Porto Alegre: Dacasa Editora, 2010, p. 13-56. 
PUELLO-SOCARRÁS, F.. Nuevo Neo-liberalismo y Administración Pública: reinvención gubernamental, post-burocracia y nueva gestión pública. Em: RODRÍGUEZ, Y. \& PUELLOSOCARRÁS, J.F. Vademecúm de la Administración Pública. Debate y Perspectivas, n. 1, p San Juan de Pasto: APESAP, 2018 a , 105-126.

PUELLO-SOCARRÁS, F.. La Economía Naranja: Otra “innovación” neoliberal para exprimirle el jugo a los trabajadores. Revista Izquierda, n. 73, p. 47-53, $2018 \mathrm{~b}$.

PUELLO-SOCARRÁS, F.. Ocho tesis sobre el neoliberalismo (1973-2013). In: RAMÍREZ, Hernán. O neoliberalismo Sul-americano em clave transnacional: enraizamento, apogeu e crise. Oikos, Unisinos, p. 13-57, 2013.

PUELLO-SOCARRÁS, F.. Nueva Gramática del Neo-liberalismo. Itinerarios teóricos, trayectorias intelectuales, claves ideológicas. Bogotá: Universidad Nacional de Colombia, Facultad de Derecho, Ciencias Políticas y Sociales, 2008.

RIFKIN, J. El fin del trabajo: Nuevas tecnologías contra puestos de trabajo - El nacimiento de una nueva era. Barcelona: Crítica, 1994

SAAD-FILHO, A.; JOHNSTON, D. Neoliberalism: A critical reader. Chicago: University of Chicago Press, 2005.

STOLOWICZ, Beatriz. EI misterio del posneoliberalismo. ILSA, Instituto Latinoamericano para una Sociedad y un Derecho Alternativos, 2016.

RETAMAR, Roberto Fernández. Nuevos primeros días. Revista de la Casa de las Américas, n. 244, p. 128-145, 2006. 